\title{
Focusing of short-pulse high-intensity laser-accelerated proton beams
}

\author{
Teresa Bartal ${ }^{1,2}$, Mark E. Foord ${ }^{2}$, Claudio Bellei' ${ }^{2}$, Michael H. Key ${ }^{2}$, Kirk A. Flippo ${ }^{3}$, \\ Sandrine A. Gaillard ${ }^{4}$, Dustin T. Offermann ${ }^{3}$, Pravesh K. Patel ${ }^{2}$, Leonard C. Jarrott ${ }^{1}$, \\ Drew P. Higginson ${ }^{1,2}$, Markus Roth ${ }^{5}$, Anke Otten ${ }^{5}$, Dominik Kraus ${ }^{5}$, Richard B. Stephens ${ }^{6}$, \\ Harry S. McLean ${ }^{2}$, Emilio M. Giraldez ${ }^{6}$, Mingsheng S. Wei ${ }^{6}$, Donald C. Gautier ${ }^{3}$ and Farhat N. Beg ${ }^{1 \star}$
}

Recent progress in generating high-energy ( $>50 \mathrm{MeV}$ ) protons from intense laser-matter interactions $\left(10^{18}-10^{21} \mathrm{~W} \mathrm{~cm}^{-2}\right.$; refs 1-7) has opened up new areas of research, with applications in radiography ${ }^{8}$, oncology ${ }^{9}$, astrophysics ${ }^{10}$, medical imaging ${ }^{11}$, high-energy-density physics ${ }^{12-14}$, and ion-proton beam fast ignition ${ }^{15-19}$. With the discovery of proton focusing with curved surfaces ${ }^{20,21}$, rapid advances in these areas will be driven by improved focusing technologies. Here we report on the first investigation of the generation and focusing of a proton beam using a cone-shaped target. We clearly show that the focusing is strongly affected by the electric fields in the beam in both open and enclosed (cone) geometries, bending the trajectories near the axis. Also in the cone geometry, a sheath electric field effectively 'channels' the proton beam through the cone tip, substantially improving the beam focusing properties. These results agree well with particle simulations and provide the physics basis for many future applications.

The ability to generate high-intensity well-focused proton beams will potentially open the door to new regimes in high-energydensity science as well as enabling a broad range of new applications. For example, an intense multi-MeV proton beam incident on solid density or compressed material can create terapascal pressures, allowing the study of the properties of warm dense matter found in the interior of giant planets such as Jupiter ${ }^{10}$. Laser-produced proton beams are also making an impact on medical applications such as isotope production ${ }^{11}$ for positron emission tomography (PET) and proton oncology ${ }^{9}$. Furthermore, energetic proton/ion beams are used to produce highly directional neutrons for applications in medicine, material science, and neutron resonance spectroscopy ${ }^{13,14}$. In the inertial fusion 'fast ignition' (FI) concept ${ }^{19}$, an intense laser generates a pulse of charged particles that ignites deuterium-tritium (DT) fuel compressed to $\approx 300 \mathrm{~g} \mathrm{~cm}^{-3}$. Although much recent work has investigated relativistic electrons as the ignitor ${ }^{22}$, proton beams, unlike intrinsically divergent electron beams, could be focused on the ignition spot and provide direct energy transport to the fuel.

In the conceptual proton FI scheme ${ }^{17}$, the proton source foil consists of a partial spherical shell placed near the end of a hollow cone which is attached to the side of a radiation cavity (hohlraum). This geometry shields the source foil from intense soft X-ray radiation generated within the hohlraum during the compression. The new work reported here builds on the proof-of-concept demonstrations of cone-in-shell compression ${ }^{23}$ without a hohlraum, where the cone acts both as a guide for the ignitor beam as well as a shield. The properties of the proton beam in this particular geometry require careful examination, especially as the viability of proton FI requires both focusing at the compressed fuel between 20 and $40 \mu \mathrm{m}$ (refs 16,18), depending on the model, and a conversion efficiency of $\approx 15 \%$ from petawatt laser pulse energy to proton beam energy ${ }^{9,18}$. Studies have shown efficiencies approaching the requirement for FI (refs 6,7,24) and proton focusing from an open geometry curved foil has been demonstrated by laser irradiation of hemispherical $\mathrm{Al}$ shells ${ }^{20,21}$. Control of divergent proton beams in flat-foil experiments has been shown using electrostatic fields when the beams pass through charged secondary ${ }^{25}$ or attached ${ }^{26}$ structures, and better control of the beam divergence has recently been reported in a cylindrical thick-foil geometry ${ }^{27}$. Here we present the first demonstration of the generation and focusing of a proton beam in a FI geometry, where the beam is generated from a curved focusing surface, which propagates and is channelled via surface fields through an enclosed cone structure, similar to that envisioned for FI targets ${ }^{23}$. The strong radial electric field from the hot-electron pressure in the proton beam and its interaction with the surrounding protective structure is shown to be critical in determining the beam dynamics near focus. This effect has not been observed previously with a non-focusing (flat-foil) source geometry and is key to understanding the curved proton trajectories in this compact proton focusing device.

Experiments were conducted on the TRIDENT subpicosecond laser at Los Alamos National Laboratory ${ }^{28}$. The proton beam was generated by irradiating a partial spherical shell of high-density carbon attached to a $60^{\circ}$ cone or cylinder structure (Fig. 1). A thin adsorbed layer of hydrocarbons on the foil surface ${ }^{29}$ provided the source of the protons. Partial and full freestanding hemispherical shell targets were also included for comparison.

The focusing characteristics of the beam were determined by imaging the protons through a $\mathrm{Cu}$ mesh and recording the mesh pattern on a stack of radiochromic film (RCF; Fig. 1). Each layer of film responds to a narrow range of proton energies at the Bragg peak, which is determined by stopping powers and the film composition $^{30}$. A three-dimensional (3D) ray tracing technique projects back the shadow of the mesh on the RCF through the original mesh, forming a bundle of converging rays. The minimum diameter $\mathrm{D}_{80}$, defined as the diameter encompassing $80 \%$ of the rays, is calculated for each type of target (Fig. 2a). A common feature

\footnotetext{
${ }^{1}$ Department of Mechanical and Aerospace Engineering, University of California-San Diego, La Jolla, California 92093, USA, ${ }^{2}$ Lawrence Livermore National Laboratory, Livermore, California 94551, USA, ${ }^{3}$ Los Alamos National Laboratory, Los Alamos, New Mexico 87545, USA, ${ }^{4}$ Helmholtz-Zentrum Dresden-Rossendorf (HZDR), 01314 Dresden, Germany, ${ }^{5}$ Institut für Kernphysik, Technische Universitat Darmstadt, 64289 Darmstadt, Germany, ${ }^{6}$ General Atomics, San Diego, California 92121, USA. *e-mail: fbeg@ucsd.edu.
} 


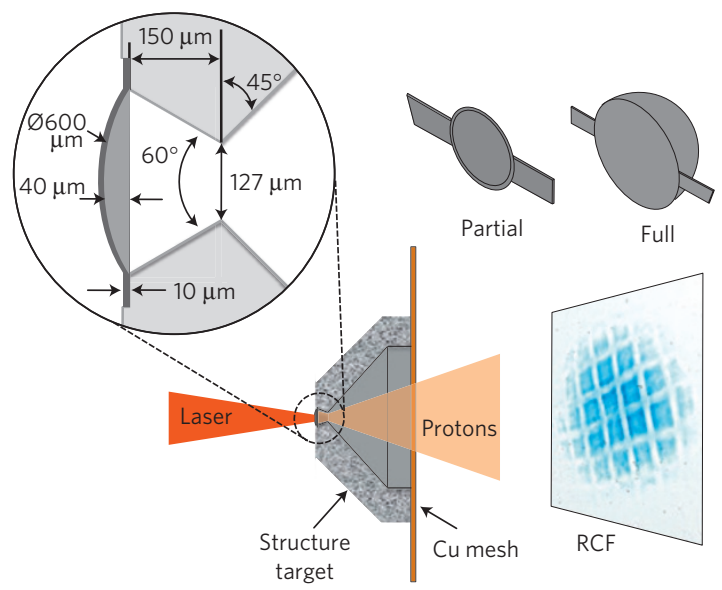

Figure 1 | Experimental set-up and targets. The cone target (expanded) consists of a 10- $\mu \mathrm{m}$-thick spherical shell, attached to the Al cone structure. A Cu mesh (200 LPI) is positioned $1.5 \mathrm{~mm}$ from the apex of the hemisphere and the RCF stack is at $4 \mathrm{~cm}$. Representative RCF data from a cone structure target is shown. The freestanding partial and full hemispherical shell targets shown were used for comparison. The cylindrical target (not shown) replaces the $60^{\circ}$ cone with a $150-\mu$ m-length cylindrical section along the same axis.

is that $\mathrm{D}_{80}$ decreases with increased proton energy. Of note, the $\mathrm{D}_{80}$ is $\approx 50 \%$ smaller for the cone and cylinder targets than for the freestanding partial and full hemispheres.

The magnification of the mesh image on the RCF pack is used to infer a focal position of the beam. For the cone target this position is located furthest from the source foil, near $z=300 \mu \mathrm{m}(z=0$ is the apex of the hemisphere), whereas for the other geometries it lies within the spherical radius of curvature, near $z=100 \mu \mathrm{m}$ (Fig. 2b). To understand these results, simulations were performed to track the particle trajectories and the evolution of the beam through the surrounding structure.

The generated proton beam was simulated using the hybrid particle-in-cell code LSP (ref. 31). The trajectories for a group of test particles that originate along the target surface at different radial positions are shown in Fig. 3. In both geometries, the majority of test protons initially accelerate normal to the surface towards the geometric centre at $z=300 \mu \mathrm{m}$, which is consistent with the 'target normal sheath acceleration' (TNSA) model ${ }^{1,3}$, where the hot electrons generated from the intense laser-matter interaction create an accelerating sheath electric field normal to the surface. After the initial acceleration, the proton trajectories do not continue in a straight line, but tend to bend away from the axis.

Further analysis of the proton simulation particles $\left(N_{\text {protons }} \approx\right.$ $\left.8 \times 10^{6}\right)$ allows a detailed comparison with the data. The protons reach their final asymptotic velocities (that is, become ballistic) at late times $(\approx 18 \mathrm{ps})$. Those trajectories are then geometrically projected back to construct a $\mathrm{D}_{80}$ diameter, analogous to the ray tracing technique applied to the RCF data. Results are compared in Fig. 4a for the freestanding target, showing similar minimum values and $\mathrm{D}_{80}(z)$ profiles. Results for the cone case (not shown) also give similar agreement with the corresponding data, providing confidence that the LSP code is accurately modelling the proton trajectories and the expansion physics in both open and enclosed (cone) geometries.

The curved proton trajectories are qualitatively explained by considering a simple model for the radial electric field generated in the proton beam. Following the initial acceleration phase near the surface ${ }^{1,3}$, the hot electrons are confined by the ambipolar field of the positively charged proton beam. The hot-electron pressure gradient sets up a radial electric field, $E_{\mathrm{r}} \approx-\nabla\left(P_{\mathrm{e}}\right) / n_{\mathrm{e}} \approx k T_{\text {ehot }} / R$, a

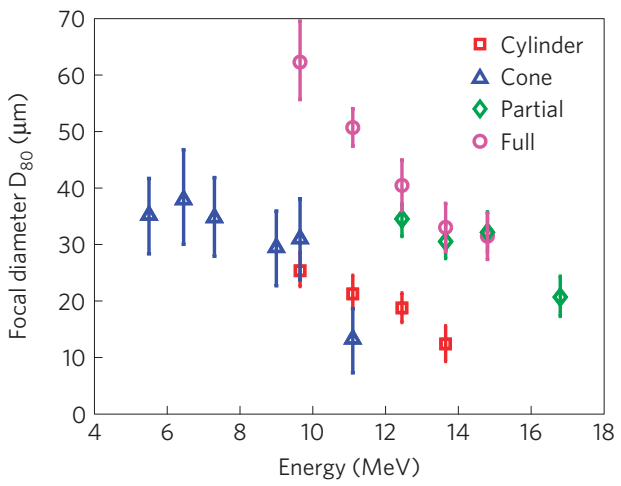

b

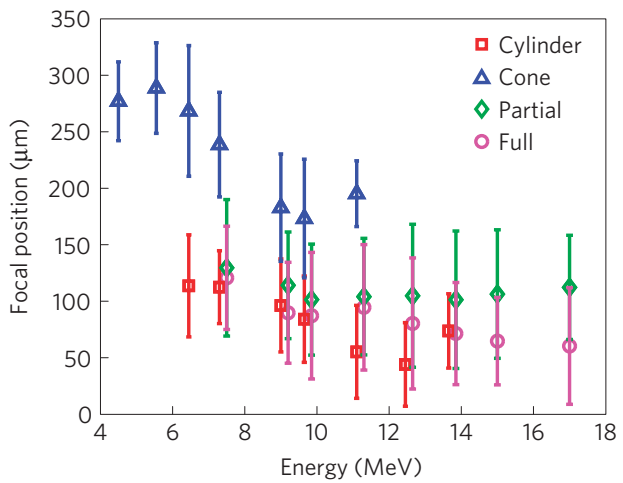

Figure $\mathbf{2} \mid \mathbf{D}_{80}$ diameter and focal position. $\mathbf{a}, D_{80}$ diameter for each represented energy (RCF layer) and target geometry determined from 3D ray tracing. Cone (blue triangles) and cylinder (red squares) enclosed geometries show significantly smaller $D_{80}$ values at most proton energies compared with the freestanding hemispherical shells. $\mathbf{b}$, Focal position of the proton beam at different proton energies, determined from the mesh magnification. For reference, the inside surface (apex) of the foil is $z=0$. The cone targets (blue triangles) have an apparent focal position that is significantly further from the apex. Error analysis is discussed in the Methods section.

where $R$ is the radial scale length of the beam, $k T_{\text {ehot }}$ is the hot-electron temperature, $P_{\mathrm{e}}$ is the hot-electron pressure and $n_{\mathrm{e}}$ is the hot-electron density. From monitoring the time history of the electric field in the frame of selected protons, the radial field switches from being directed inwards to directed outwards, as the radial field from the hot electrons surpasses the radial acceleration force that dominates near the surface. We note that the weak scaling of the radial electric fields with density $\left(E_{\mathrm{r}} \approx k T_{\text {ehot }} / R\right)$ suggests that the focusing should not be substantially degraded for high-current-density beams, such as required for proton FI. Higher laser intensities, which will generate higher-energy electrons, will increase the radial pressure to some extent, although this scales weakly with laser intensity $\left(T_{\text {ehot }} \sim I_{\mathrm{L}}^{0.5}\right)$.

Simulations indicate that the radial field in the beam is of the order of a few $\mathrm{MV} / 100 \mu \mathrm{m}$, which is sufficient to deflect a multi$\mathrm{MeV}$ proton over the spatial scale of the target. It is also interesting to note that this heuristic model predicts that higher-energy protons should penetrate to smaller radii before bending, which is the trend in the data seen in Fig. 2a, consistent with recently reported carbon ion-beam experiments ${ }^{32}$.

The inferred focal position near $z=100 \mu \mathrm{m}$, as shown in Fig. 2b, is understood by considering the diagnostic method. Both the ray tracing and magnification methods inherently assume straight-line proton trajectories. Depending on the curvature of the trajectories, the inferred focal position determined from extrapolating the trajectories to the axis may fall much inside the actual focal position of the proton beam. Therefore, the time-dependent proton 
a

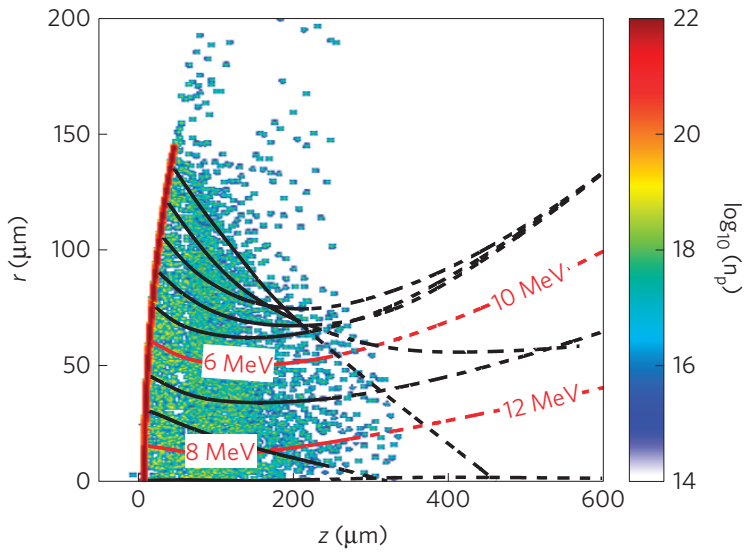

b

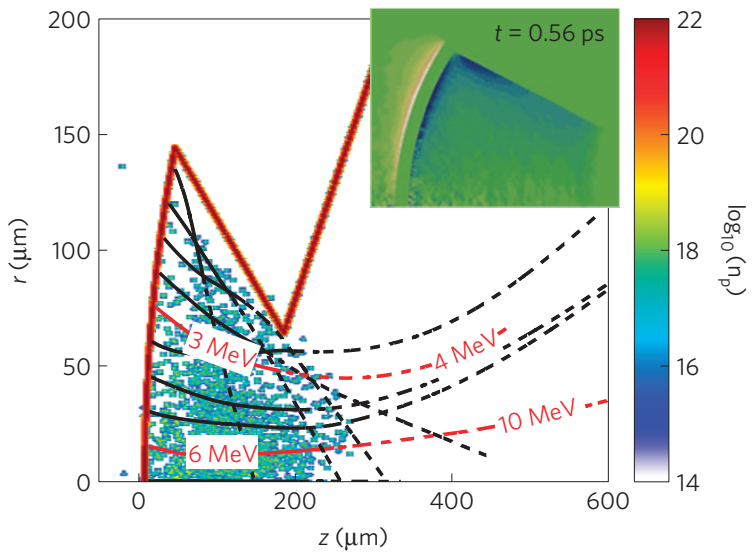

Figure $\mathbf{3}$ | Simulation of probe particles. $\mathbf{a}, \mathbf{b}$ Proton density maps at $t=7.3 \mathrm{ps}$ for the case of a partial hemisphere without a surrounding structure (a) and with a surrounding cone structure (b). Note that the radial scale is expanded. For both cases, the trajectories of test proton particles are also shown, with solid lines to $t<7.3 \mathrm{ps}$ and broken lines from $7.3 \mathrm{ps}<t<19.2 \mathrm{ps}$. For comparison, in each plot the kinetic energy gained by two sample particles is also shown (in red), where more energetic protons are emitted closer to the axis. $\mathbf{b}$ (inset), Spatial distribution of the radial electric field component for the cone target at $0.56 \mathrm{ps}$. Dark blue scale is negative (radially inwards), light yellow/white is positive (radially outwards).

distributions from the simulations are used to calculate the fluence profiles $\Delta_{80}(z)$, defined as the time-integrated flux diameter that encompasses $80 \%$ of the protons through a plane at position $z$, which would more correctly represent the focal position and diameter of the beam. The fluence profiles $\Delta_{80}(z)$ for protons with energy $>3 \mathrm{MeV}$ are plotted in Fig. $4 \mathrm{~b}$, corresponding to the approximate energy required for FI deposition ${ }^{18}$. For the freestanding target, the fluence diameter is $\Delta_{80} \approx 90 \mu \mathrm{m}$, which is much larger than the more peaked $\mathrm{D}_{80}$ profiles seen in Fig. $4 \mathrm{a}$. As for the cone target, the fluence diameter is significantly reduced to $\Delta_{80} \approx 60 \mu \mathrm{m}$. The reduced $\Delta_{80}$ diameter is the result of a sheath electric field that develops along the inside surface of the cone wall, generated from the hot-electron sheath that extends upward from the laser spot radius (Fig. $3 \mathrm{~b}$ inset). This field bends the protons that propagate near the wall surface (Fig. 3b). The sheath field is strongest during the initial phase of focusing, as the proton beam begins to propagate through the cone. At later times, the field is reduced as the hydrocarbon layer from the surface begins to expand. The sheath field effectively channels the protons through the tip of the cone, extending the focal position. As shown in Fig. $2 b$, this effect is not observed for the other geometries, where the wall focusing is not present (or less effective for the cylinder case) and

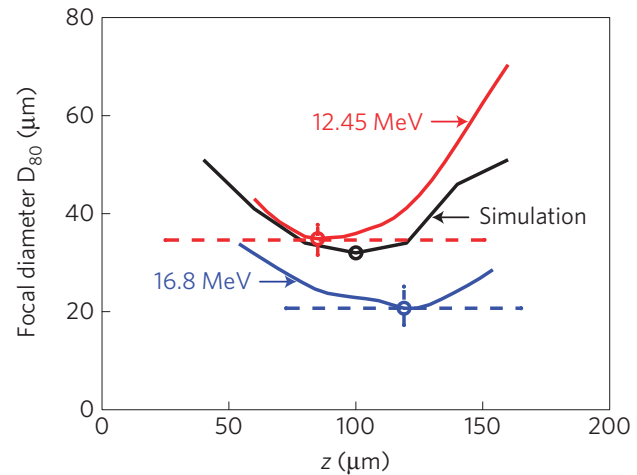

b

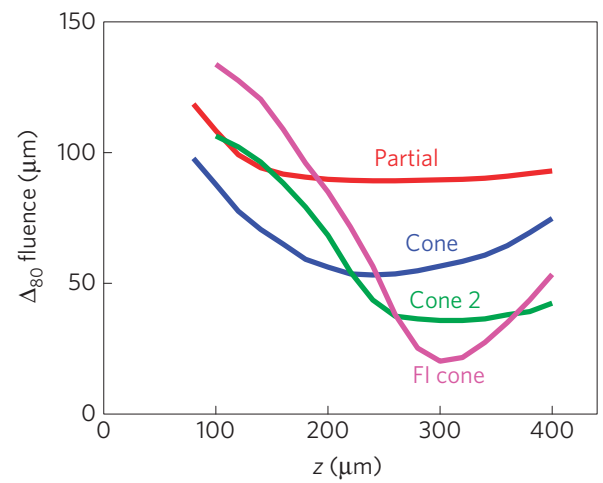

Figure $4 \mid D_{80}$ and $\Delta_{80}$ fluence profiles. a, Comparison of experimental and simulation results for a freestanding partial hemisphere target. The profile of $\mathrm{D}_{80}(z)$ is plotted. The circles, along with the appropriate error bars, represent the minimum $D_{80}$. The simulation includes all protons with $E>9 \mathrm{MeV}$. b. Fluence curves $\Delta_{80}(z)$ for proton energies $>3 \mathrm{MeV}$ for the partial hemisphere and the cone target. Also shown are simulation results with a uniformly illuminated cone target (cone 2), and with a uniformly illuminated thin-walled cone Au target (FI cone), as described in the text.

the radial hot-electron pressure in the beam expands the beam at a closer distance to the apex of these targets.

The beam focusing also depends on the spatial uniformity of the hot-electron source. Hot electrons generated in the intense laser spot region (laser spot diameter $\approx 90 \mu \mathrm{m}$ ) propagate transversely along the surface, creating a hot-electron radial pressure gradient. Expanding the hot-electron source width from 90 to $360 \mu \mathrm{m}$ reduced the initial radial gradient of the hot-electron sheath and resulted in a more convergent beam, with $\Delta_{80}$ reduced from 60 to $35 \mu \mathrm{m}$ (Fig. 4b, cone 2). Further simulations with a uniform source, but with no hydrocarbon layer on the cone wall, generated a focused beam with $\Delta_{80} \approx 20 \mu \mathrm{m}$ (Fig. $4 \mathrm{~b}$, FI cone). In this case, focusing is enhanced owing to the lack of a thin hydrocarbon layer, which reduces the sheath field as it expands. We expect this case to be applicable for the much higher current conditions required for FI, where a low-density hydrocarbon layer on the cone wall would be a relatively small perturbation. On the basis of these results, improvements in the laser uniformity and optimizing the curvature and surrounding focusing structure may allow even higher focused beam intensities to be achieved, which have direct applications in highenergy-density science, such as in astrophysics ${ }^{10}$ and FI research, as well as in the medical and nuclear applications discussed above.

\section{Methods}

Experiments were conducted on the 200 TW TRIDENT short pulse laser at Los Alamos National Laboratory, which delivered 70-80 J on target in 500-600 fs with an amplified spontaneous emission contrast ratio better than $10^{-9}$ (ref. 28). The $1,053 \mathrm{~nm}$ laser pulse was focused by an $f / 8$ parabolic mirror, which produced a spot size containing $50 \%$ of the energy in a diameter of $90 \mu \mathrm{m}$. The hemispherical shells were made from high-density carbon by a chemical vapour deposition 
process. The shells were $10 \mu \mathrm{m}$ thick with a radius of curvature of $300 \mu \mathrm{m}$, with the partial shells having a chord length of $300 \mu \mathrm{m}$. The surrounding structure was $\mathrm{Al}$ and the $\mathrm{Cu}$ mesh, of 200 lines per inch (LPI), was glued on the rear side at a distance of approximately $1.5 \mathrm{~mm}$ from the apex of the hemispherical shell, with its position measured to within $\pm 20 \mu \mathrm{m}$

Radiochromic film is a dosimetry film with an active layer that undergoes a chemical reaction when exposed to ionizing radiation ${ }^{30}$. The chemical reaction darkens the film in proportion to the radiation dose. In the experiment, a stack of RCF containing $4 \mathrm{~cm} \times 4 \mathrm{~cm}$ squares of film alternated with aluminium filters $(100 \mu \mathrm{m}-1.5 \mathrm{~mm}$ thick) was placed $4 \mathrm{~cm} \pm 500 \mu \mathrm{m}$ behind the target normal. The film was scanned using a flatbed scanner to capture the image of the proton beam.

In the $3 \mathrm{D}$ ray tracing technique, straight-line proton trajectories were reconstructed by connecting the mesh intersection points on the RCF to the intersection points on the original mesh. The resulting ray bundle was used to determine the $\mathrm{D}_{80}$ diameter and position. The calculation of $\mathrm{D}_{80}$ is dependent on accurately choosing the mesh intersection points on the RCF and adequately representing the whole proton beam by the selection of the points. For the freestanding targets, in the RCF layers corresponding to the lower-energy protons, portions of the mesh image on the RCF were distorted, which prevented the use of the entire beam; therefore, the focal spot size for the lower-energy protons could not be calculated. The focal position was calculated using the measured mesh magnification on the RCF and geometrical considerations. To calculate the mesh magnification, the distance between the mesh intersection points on the RCF were measured and divided by the actual mesh size, then the average was taken. The error in the magnification is represented by the standard deviation in the measurement. The number of mesh intersection points selected varies from shot to shot and the RCF layer of interest, ranging from 10 to 80 . The overall error in the calculation takes into account the error in the measured distances in the target set-up along with the standard deviation in determining the mesh magnification. The calculated focal positions using either ray tracing or geometrical considerations were within the calculated error.

The $\mathrm{D}_{80}$ diameter encompassing $80 \%$ of the rays is determined using a bootstrap method. At a given plane along the longitudinal direction $(z)$, the $3 \mathrm{D}$ ray tracing technique provides the position of $N$ rays (where $N$ is the number of chosen mesh intersections on the RCF film) along with their uncertainties. After assuming that each experimental data point is a realization from a Gaussian probability density function, an overall empirical probability density function is constructed. A Monte Carlo sampling without replacement is then applied: $N$ samples are randomly chosen from the probability density function, making sure that exactly one data point is chosen in the uncertainty region that surrounds each experimental data point. This sampling is then repeated over $k$ realizations, where $k>10^{4}$. A distribution function for $\mathrm{D}_{80}$ is obtained with a determination at the $95 \%$ confidence level.

LSP (ref. 31) is a hybrid particle-in-cell code used to simulate the generation and focusing of the proton beam in the various experimental geometries. Here two-dimensional cylindrical symmetry is assumed. Relativistic electrons were converted from cold background electrons in the target, simulating the generation of hot electrons from an intense laser interaction with a solid. The electron source has a spatial Gaussian width consistent with the laser spot diameter $(\approx 90 \mu \mathrm{m})$ and its duration matches the laser pulse length. The electrons are directed into the target with a $T_{\mathrm{e}}=600 \mathrm{keV}$ relativistic Jüttner distribution with a forward drift of $\gamma \beta_{z}=0.6$, which results in a $45^{\circ}\left(30^{\circ}\right)$ forward half angle containing $50 \%$ of the energy of all electrons (those with $E>5 \mathrm{MeV}$ ), respectively. This distribution was chosen to be consistent with the maximum proton energies observed for the cone and partial hemispherical targets, found to be $11 \mathrm{MeV}$ and $17 \mathrm{MeV}$, respectively. The simulations ran for approximately $18 \mathrm{ps}$, which was required for the protons to reach asymptotic velocities.

\section{Received 8 March 2011; accepted 26 October 2011; published online 4 December 2011}

\section{References}

1. Hatchett, S. P. et al. Electron, photon, and ion beams from the relativistic interaction of Petawatt laser pulses with solid targets. Phys. Plasmas 7, 2076-2082 (2000).

2. Snavely, R. A. et al. Intense high-energy proton beams from petawatt-laser irradiation of solids. Phys. Rev. Lett. 85, 2945-2948 (2000).

3. Wilks, S. C. et al. Energetic proton generation in ultra-intense laser-solid interactions. Phys. Plasmas 8, 542-549 (2001).

4. Borghesi, M. et al. Multi-MeV proton source investigations in ultraintense laser-foil interactions. Phys. Rev. Lett. 92, 055003 (2004).

5. Fuchs, J. et al. Laser-driven proton scaling laws and new paths towards energy increase. Nature Phys. 2, 48-54 (2006).

6. Hegelich, B. M. et al. Laser acceleration of quasi-monoenergetic $\mathrm{MeV}$ ion beams. Nature 439, 441-444 (2006).
7. Robson, L. et al. Scaling of proton acceleration driven by petawatt-laser-plasma interactions. Nature Phys. 3, 58-62 (2007).

8. Mackinnon, A. J. et al. Proton radiography of a laser-driven implosion. Phys. Rev. Lett. 97, 045001 (2006).

9. Bulanov, S. V. \& Khoroshkov, V. S. Feasibility of using laser ion accelerators in proton therapy. Plasma Phys. Rep. 28, 453-456 (2002).

10. Baraffe, I. The structure and evolution of giant planets. Space Sci. Rev. 116, 67-76 (2005).

11. Fritzier, S. et al. Proton beams generated with high-intensity lasers: Applications to medical isotope production. Appl. Phys. Lett. 83, 3039-3041 (2003).

12. Dyer, G. M. et al. Equation of state measurements of dense plasma heated with fast protons. Phys. Rev. Lett. 101, 015002 (2008).

13. Mason, T. E. Pulsed neutron scattering for the 21st century. Phys. Today 59, 44-49 (May, 2006)

14. Higginson, D. P. et al. Laser generated neutron source for neutron resonance spectroscopy. Phys. Plasmas 17, 100701 (2010).

15. Key, M. H. et al. Study of electron and proton isochoric heating for fast ignition. J. Phys. IV France 133, 371-378 (2006).

16. Key, M. H. et al. Proton fast ignition. Fusion Sci. Tech. 49, 440-452 (2006).

17. Roth, M. et al. Fast ignition by intense laser-accelerated proton beams. Phys. Rev. Lett. 86, 436-439 (2001).

18. Temporal, M. \& Honrubia, J. J. Numerical study of fast ignition of ablatively imploded deuterium-tritium fusion capsules by ultra-intense proton beams. Phys. Plasmas 9, 3098-3107 (2002).

19. Tabak, M. et al. Ignition and gain with ultrapowerful lasers. Phys. Plasmas 1, 1626-1634 (1994)

20. Snavely, R. A. et al. Laser generated proton beam focusing and high temperature isochoric heating of solid matter. Phys. Plasmas 14, 092703 (2007).

21. Patel, P. K. et al. Isochoric heating of solid-density matter with an ultrafast proton beam. Phys. Rev. Lett. 91, 125004 (2003).

22. Green, J. S. et al. Surface heating of wire plasma using laser-irradiated cone geometries. Nature Phys. 3, 853-856 (2007).

23. Kodama, R. et al. Fast heating of ultra-high density plasma as a step toward laser fusion ignition. Nature 412, 798-802 (2001).

24. Hey, D. S. et al. Laser-accelerated proton conversion efficiency thickness scaling. Phys. Plasmas 16, 123108 (2009).

25. Toncian, T. et al. Ultrafast laser- driven microlens to focus and energy-select mega-electron volt protons. Science 312, 410-413 (2006).

26. Kar, S. et al. Dynamic control of laser-produced proton beams. Phys. Rev. Lett. 100, 105004 (2008)

27. Kar, S. et al. Ballistic focusing of polyenergetic protons driven by petawatt laser pulses. Phys. Rev. Lett. 106, 225003 (2011)

28. Batha, S., Aragonez, R. \& Archuleta, F. TRIDENT high-energy-density facility experimental capabilities and diagnostics. Rev. Sci. Instrum. 79, 10F305 (2008).

29. Foord, M. E. et al. Proton generation and efficiency from an intense laser irradiated foil. High Energy. Density Phys. 3, 365-370 (2007).

30. Nürnberg, F. et al. Radiochromic film imaging spectroscopy of laser-accelerated proton beams. Rev. Sci. Instrum. 80, 033301 (2009).

31. Welch, D. R., Rose, D. V., Oliver, B. V. \& Clark, R. E. Simulation techniques for heavy ion fusion chamber transport. Nucl. Instrum. Methods A 464, 134-139 (2001).

32. Offermann, D. T. et al. Characterization and focusing of light ion beams generated by ultra-intensely irradiated thin foils at the kilojoule scale. Phys. Plasmas 18, 056713 (2011).

\section{Acknowledgements}

The authors sincerely thank P. Norreys for helpful discussions concerning this work and gratefully acknowledge the support of the staff at the TRIDENT laser facility at Los Alamos National Laboratory. We would also like to thank T. Yabuuchi for useful discussions. T.B. is supported through the Lawrence Scholar Program at Lawrence Livermore National Laboratory. This work was performed under the auspices of the US Department of Energy by Lawrence Livermore National Laboratory under Contract DE-SC0001265. M.R., A.O. and D.K. are supported by the BMBF 06DA9044I.

\section{Author contributions}

F.N.B., M.E.F., P.K.P., R.B.S., M.H.K., H.S.M., E.M.G. M.S.W. and T.B. were involved in the project planning and target design. T.B., K.A.F., D.T.O., S.A.G., L.C.J., D.P.H., D.C.G., A.O., D.K. and M.R. contributed to the experimental work. T.B. and C.B. carried out the data analysis and wrote the letter along with F.N.B. and M.E.F., where M.E.F. performed the simulations.

\section{Additional information}

The authors declare no competing financial interests. Reprints and permissions information is available online at http://www.nature.com/reprints. Correspondence and requests for materials should be addressed to F.N.B. 